\title{
science \\ Scientific journals should be transformed into science storytellers to improve their visibility
}

\author{
Kwangil Oh \\ Editage, Cactus Communications Korea, Seoul, Korea
}

\begin{abstract}
What is the objective for science journals to publish research papers? Would it be enough to collect research manuscripts and simply publish them in print or on the web? Science journal publishers have always strived to find ways of disseminating journal content to as many readers as possible. It is now time for science journal publishers to think about why a journal should be published; whether it is acceptable for valuable scientific findings to lie dormant in a journal's archive; and whether traditional science communication is still effective. The present article suggests that science journals should transform themselves into science storytellers to improve the visibility and discoverability of their research findings. First, a new communication network between journals, authors, peers, the public, and policymakers is required. Second, conversion of media from academic language to plain language is critical to broadening the audience. Third, audio-visual content should be introduced into journal publishing to facilitate easy comprehension of the content. Fourth, research-focused channels, including EurekAlert, Medium, and social networking service channels are recommended as new media to propagate journals' content to researchers. Improving visibility and discoverability is an urgent mission, especially for small society journals. To achieve this mission, science journals should be adapted to become storytellers and science communicators, as suggested above. A small society journal's editor is not merely an editor, but an editor-publisher; therefore, editors should understand and take on this role.
\end{abstract}

Keywords

Science communication; Journal visibility; Publications; Science storytelling; Social networking

Received: July 29, 2021

Accepted: August 2, 2021

Correspondence to Kwangil Oh kwangil@cactusglobal.com

ORCID

Kwangil Oh

https://orcid.org/0000-0002-8129-8683

\section{Introduction}

Background/rationale: What image do people have in their mind when they think of a "science journal"? Interestingly, this image may not be clear. The word "journal" is defined in the $O x$ ford learner's dictionary of academic English as "a newspaper or magazine that deals with a par- 
ticular subject or profession"[1]. People may easily assume that science journals deal with various subjects in the domain of science. Here, one may ask-what images of "science" do they have? Does a picture of a physicist such as Einstein come to mind? Does a complicated equation strike them? They may think of a scientific novel that they read in childhood-or, potentially, a scientific-themed movie such as "The Terminator" or "Interstellar." The image of science might determine a person's attitudes toward "science journals." A boring image of science will result in the prejudice that a "science journal" would be boring.

Journal publishers are often concerned that their journals may fail to attract the public's interest. Therefore, they are increasingly faced with the need to solve the problem of finding ways to improve the visibility or discoverability of their journals. People's belief that scientific articles are difficult to read is a major challenge hindering efforts to improve the public's understanding of science. This issue also shapes the communication methods used to deliver journal content to readers, and science journals should find a way to successfully "deliver" their content to an audience that goes beyond their traditional readership.

The time has come for us to solve this challenge and move forward to a new age of science communication. In this new paradigm, journal publishers should shift their identity to science storytellers. Science communicators can be defined as people from any background who communicate about various science-related topics; this category encompasses non-fiction authors, journalists, bloggers, news editors, and beyond [2]. Science communicators can be seen as storytellers delivering science to a lay audience in easy-to-understand language. If they understand the values of the scientific community, and the interests and values that readers perceive, their storytelling will serve as a bridge between scientists and the public [2].

Objectives: This essay suggests that science journals should transform into science storytellers to improve the visibility and discoverability of their research findings.

\section{What is the New Science Communication Process?}

Successful science communicators use appropriate skills, media, activities, and dialogue to induce responses such as awareness, enjoyment, interest, opinions, and understanding among their audience [3]. The goal of science communication is for the audience to become aware of new scientific findings, enjoy the content, become interested enough to engage with science and its communication; form science-related opinions or attitudes, and understand scientific content and social factors [3].

Journals' traditional way of communicating with authors and readers was to receive manuscripts from scientists and publish those manuscripts in print. Readers, most of whom were scientists or researchers working in a related field, then read the printed content. This framework was limited to oneway communication in a single format (text), with minimal participation by readers. Traditional science communication is still influential in scientific journal publishing. However, the internet has changed scientific journal publishing, and electronic journals have become more common than ever. Readers can access electronic versions of research articles wherever they are located. Therefore, we can say that journal articles are "consumed" rather than being "read."

Next, publishers may wonder, "How should science communication develop in this new environment?" Journals should utilize various media to deliver scientific content and findings to a wider audience multi-directionally in multiple formats. Interactional communication will encourage the audience to share their interest in scientific findings with other people and to form opinions on the content. The aim of this communication process is to create a virtuous circle between

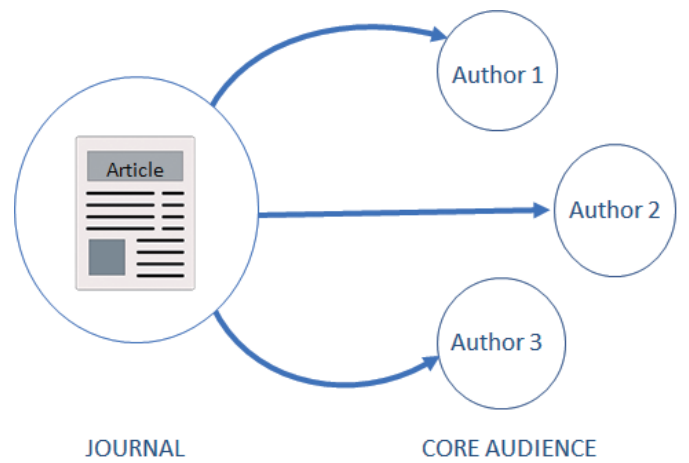

- One-way communication

- Single-format content

- Limited engagement with wider audience

Fig. 1. Diagram of traditional science communication. Reproduced from Calamur H. Traditional science communication [Unpublished internal material]. Mumbai: Cactus Communications; 2021, with permission from Cactus Communications [4]. 
a journal publisher, scientists, and the audience. Fig. 1 [4] and Fig. 2 [5] illustrate how the new paradigm of science communication can be different from traditional science communication.

\section{Media Conversion}

The main objective of new science communication is to deliver content presenting new findings, scientists' passion for research, and problem-solving through science, with the ultimate goal of answering the question of how science will change our life. How do science communicators achieve this goal? Above all, they must utilize various media and formats through a process that will be called "media conversion." It should be noted that the act of transcribing scientific findings on paper is also a type of media conversion. In the current context, we need to convert print content to other media formats. Table 1 summarizes examples of media conversion.

\section{Plain-language Summaries}

Scientific findings should be presented in a format that the public can understand easily. Scientists and laypeople use dif-

Table 1. Examples of media conversion

\begin{tabular}{ll}
\hline Original format & \multicolumn{1}{c}{ Converted format } \\
\hline Academic language & Simple language \\
Full manuscript & Summary of a manuscript \\
Full manuscript & Visual content (infographics, video summary) \\
Full manuscript & Audio content (audio summary) \\
\hline
\end{tabular}

ferent language; hence, summarizing a paper in simple, jargon-free language is the first step of media conversion. A plain-language summary aims to make scientific content easily accessible to the public, engage with a broader audience, and cross language barriers. This summary provides a foundation to convert a scientific manuscript into various formats. Four practical tips to write an attractive plain-language summary are presented as follows: (1) use simple terms; (2) avoid using sophisticated language and complex sentences; (3) do not exaggerate the implications; and (4) include all the essential findings to help readers understand the full paper.

Once a summary is created, publishers can make it easy for the public to find the summary. The following are four tips to improve the discoverability and readership of an article. (1) Write a new title optimized for search engines; (2) include graphics and artwork in the summary; (3) insert subheadings with keywords; and (4) use the summary as a basis for additional content such as social media and video summary

\section{Cross-channel Communication}

Multiple communication channels should be used to deliver scientific content to a wider audience. Having various channels is sometimes more effective than media conversion, since communication channels can form connections between a journal, scientists, and the audience. Next, shall we find channels that we can use? I would like to introduce a cross-channel communication strategy through which different channels can work together without strict boundaries. Journals can create channels for the public, researchers, and social networking services.

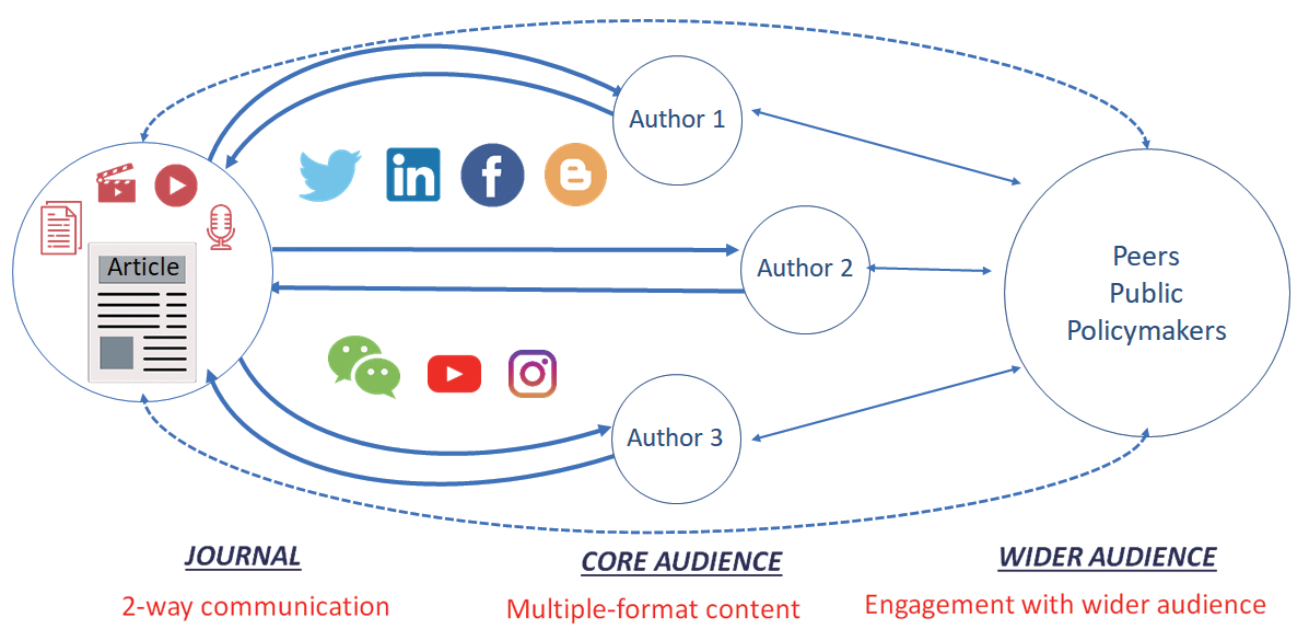

Fig. 2. Diagram of new science communication. Reproduced from Calamur H. New science communication [Unpublished internal material]. Mumbai: Cactus Communications; 2021, with permission from Cactus Communications [5]. 


\section{Public Channels}

Public channels are places, such as news portals, where journals can approach ordinary people directly. Unfortunately, publishing journal content through public channels can be surprisingly difficult. Hence, we need a channel to attract science journalists' attention. Direct communication with science journalists may be one approach; also, hiring news agencies such as Newswire may be another option. These agencies provide a press release distribution service, which helps to increase the likelihood of content receiving exposure to the wider public.

\section{Research-focused Channels}

Beyond public channels, journals need to have more focused channels that researchers and scientists are more likely to be drawn to, such as EurekAlert and Medium. EurekAlert is a research news portal with a favorable reputation, where journal publishers in all disciplines of research can release news articles upon payment of an annual subscription fee. Annual subscribers can post unlimited news articles during the 12-month period of their subscription [6]. Medium is an open platform where anyone can tell a story free of charge, and it aims to create a new model for digital publishing [7]. Journal publishers can transform into creative storytellers who present their superb insights for the benefit of humankind.

\section{Social Network Service Channels}

Social network service (SNS) channels provide an opportunity for journals to communicate with a broad audience interactively. SNS platforms have recently been developed to pro- vide places where people can share information and opinions with others, beyond simply sharing updates from their personal life. Massive amounts of user-generated content are shared through YouTube, and LinkedIn can be thought of as a professional equivalent of Facebook. Table 2 shows exemplary SNS channels of three international journal publishers. These publishers tell their stories through these channels, which will become another archive of research findings.

Twitter is another tool that attracts an audience through short, eye-catching messages, and it has become a popular communication platform for researchers. Researchers believe that Twitter citations can reflect scholarly impact faster than traditional citations, as $40 \%$ of Twitter citations occur within a week [8]. Table 3 summarizes exemplary uses of SNS as a science communication format.

\section{Alternative Metrics}

Journals often wonder about how well they are working as science communicators and the impact of their content. It would be very helpful for them to have analytical tools to measure the impact of their content on society and their audi-

Table 3. Exemplary uses of SNS for science communication

\begin{tabular}{ll}
\hline SNS type & \multicolumn{1}{c}{ Format } \\
\hline Twitter & Short summary of a paper \\
Instagram & Catchy image with a post \\
Facebook & Graphical abstract, post about the paper \\
Linkedln & Summary of the paper, accompanied by a link \\
YouTube & Slide show, video summary, video interview with authors \\
Blog & Summary of a paper in non-technical, simple language \\
\hline
\end{tabular}

SNS, social network service.

Table 2. Exemplary SNS channels

\begin{tabular}{ll}
\hline Journal publisher & \multicolumn{1}{c}{ SNS channel } \\
\hline Elsevier & YouTube: https://www.youtube.com/channel/UCnfPOvdkVXD3mIZOa4EiNjA \\
& Linkedln: https://www.linkedin.com/company/elsevier/ \\
& Facebook: https://www.facebook.com/search/top?q=elsevier \\
& YouTube: https://www.youtube.com/channel/UClbD6EDPsFkZFOXVfikRdI0 \\
Springer Nature & Linkedln: https://www.linkedin.com/company/springernaturetechnologyandpublishingsolutions/ \\
& Facebook: https://www.facebook.com/SpringerNature \\
& YouTube: https://www.youtube.com/user/channelplosone \\
& Linkedln: https://www.linkedin.com/company/public-library-of-science/ \\
& Facebook: https://www.facebook.com/PLOS.org
\end{tabular}

SNS, social network service. 
ence. Unfortunately, the traditional citation-based metrics are not able to provide these insights. Alternative metrics are also called altmetrics to emphasize their difference from bibliometrics [9]. These new metrics can be used as options to estimate the social impact of journal content, and constitute a new way to measure public engagement with research findings [9]. These are complementary metrics that can be used with citation-based metrics by measuring the interest of the audience in journal content around the world [10]. The Altmetric service run by Digital Science and Research Solutions is an example of alternative metrics that many international journal publishers have adopted. It provides numerical data and visualizes sources from the internet to show how much attention journal content is receiving and the sources of attention using the Altmetric donut [10].

\section{Conclusion}

Many small society journals still seem to operate a simple one-way communication channel to publish manuscripts on time. Most of the participants in this communication process are researchers or members of the academic society that publishes the journal. Improving the visibility and discoverability of science journals is emerging as an urgent mission. To achieve this mission, journal editors should adapt to become storytellers and science communicators. In this new age of science communication, journals should develop various communication channels to disseminate newly converted contents in multiple formats with the public. Unlike journal editors of large commercial publishing companies, a small society journal's editor is not merely an editor, but an editorpublisher. They should catch on to this new change in the environment and adopt the suggestions outlined above for journal publishing.

\section{Conflict of Interest}

No potential conflict of interest relevant to this article was reported.

\section{Funding}

The author received no financial support for this study

\section{References}

1. Lea D, Bull V, Webb SS, et al. Oxford learner's dictionary of academic English. Oxford: Oxford University Press; 2014.

2. Brown P, Scholl R. Expert interviews with science communicators: how perceptions of audience values influence science communication values and practices [version 1; peer review: 2 approved with reservations]. F1000Research 2014; 3:128. https://doi.org/10.12688/f1000research.4415.1

3. Burns TW, O’Connor DJ, Stocklmayer SM. Science communication: a contemporary definition. Public Underst Sci 2003; 12:183-202. https://doi.org/10.1177/09636625030122004

4. Calamur H. Traditional science communication [Unpublished internal material]. Mumbai: Cactus Communications; 2021.

5. Calamur H. New science communication [Unpublished internal material]. Mumbai: Cactus Communications; 2021.

6. EurekAlert. EurekAlert! Services [Internet]. Washington, DC: American Association for the Advancement of Science; 2021 [cited 2021 Jul 25]. Available from: https:// www.eurekalert.org/services

7. Medium. Every idea needs a Medium [Internet]. San Francisco, CA: Medium; 2021 [cited 2021 Jul 25]. Available from: https://medium.com/about?autoplay=1

8. Priem J, Costello KL. How and why scholars cite on Twitter. Proc Am Soc Inf Sci Technol 2020;47:1-4. https://doi. org/10.1002/meet.14504701201

9. Bornmann L. Do altmetrics point to the broader impact of research? An overview of benefits and disadvantages of altmetrics. J Inf 2014;8:895-903. https://doi.org/10.1016/ j.joi.2014.09.005

10. Altmetric. What are altmetrics? Capturing the online attention surrounding scholarly content [Internet]. London: Digital Science and Research Solutions; 2021 [cited 2021 Jul 25]. Available from: https://www.altmetric.com/aboutaltmetrics/what-are-altmetrics/ 